\title{
A Roller Coaster Project as Part of an Undergraduate Dynamics Course in Mechanical Engineering
}

\section{Dr. Andrew R. Sloboda, Bucknell University}

Andrew Sloboda is a Visiting Assistant Professor at Bucknell University where he teaches a variety of mechanics-based courses, including solid mechanics, fluid mechanics, dynamics, system dynamics, and vibration. His research interests lie primarily in the fields of nonlinear dynamics, vibration, and fluidstructure interaction. 


\title{
A Roller Coaster Project as Part of an Undergraduate Dynamics Course in Mechanical Engineering
}

\begin{abstract}
Undergraduate dynamics courses in mechanical engineering are typically structured around students solving numerous textbook-style problems in order to increase their proficiency at analyzing different scenarios involving dynamics concepts. However, students may not see how the same concepts can be useful in open-ended, design-oriented settings. To remedy this shortcoming and to help students synthesize material from different topics within dynamics, a roller coaster design project has been developed and incorporated into an undergraduate dynamics class.
\end{abstract}

This roller coaster project allows students to investigate and creatively apply their analytic skills to an ambiguous, real-world problem that they are highly motivated to explore. It both reinforces the underlying curriculum and also helps students develop intellectually, as the project is designed to teach that dynamics isn't so much about looking for the "right answer" as it is about choices and simplifications made in modeling reality.

Although roller coaster design projects have been used as the basis for entire undergraduate courses and also in STEM activities for pre-college students, the author is unaware of a similar project being included as part of a first course in dynamics. For this project, students in teams of three were tasked with designing, analyzing, and simulating a roller coaster over the course of a semester during one of the four instructional hours allotted each week. This paper details the organization of the project and the methods by which the students were assessed. It also includes data regarding student perceptions of the project, specifically how the project helped them learn to apply basic dynamics concepts, work in teams, and develop analytic skills valuable outside of the classroom.

\section{Introduction}

Instruction in an introductory mechanical engineering dynamics course is typically focused on key concepts and applying these concepts to different types of problems. The goal is to dispel misconceptions and develop in students the knowledge, intuition, and skills necessary to analyze particle and rigid body motion. The need for practice and for exposure to a wide variety of problems in order to ensure student competence means typical classes center on solving textbook-style problems. However, focusing the bulk of instructional time on such problems is dangerous because students may not see the relevance of dynamics to design tasks and to the real world. This may lead to unfortunate consequences, including a lack of motivation for learning the material, a sense that dynamics has few important engineering applications, and the idea that the process of building a mathematical model is less important than finding the "right answer."1

Projects in dynamics courses can help alleviate these issues. Projects give students the opportunity to work on larger, design-based problems that require them to integrate different dynamics-related concepts and skills and apply them in ways that are more realistic than in the 
typical textbook problem. Within this context, it is also much easier to explore the idea of general mathematical modeling, and how the choices and simplifications made in modeling have ramifications to practical issues of design and manufacture. Several undergraduate dynamics projects have been used successfully for this purpose in the past. ${ }^{2-4}$

One dynamics subject that tends to excite a large proportion of the undergraduate mechanical engineering student population is roller coaster design. This subject has been used as the basis for entire undergraduate courses ${ }^{5}$ and also in STEM activities for pre-college students. ${ }^{6}$ Roller coaster design is a rich environment to explore different aspects of particle dynamics because so many of the topics in a typical undergraduate curriculum are featured in roller coaster dynamics. Also, as a student's analytic and numerical skills improve, different levels of analysis and design become possible, and thus a roller coaster design project can be tailored to different subject matter emphases and different course lengths.

This paper presents a roller coaster project incorporated as part of a junior-level, introductory dynamics course. The project was in addition to assignments, quizzes, and exams and counted for $15 \%$ of the overall course grade. Students were placed in teams of three by the instructor after providing the names of two colleagues with whom they would prefer to work and two colleagues with whom they would prefer not to work. Each team was then given the opportunity to design a roller coaster and simulate it using the commercially available roller coaster simulation program NoLimits during one of the four instructional hours each week. The project was designed to reinforce the conceptual understanding and analytic skills being developed in the lecture portion of the course, while also demonstrating that what was being learned was valuable in a design setting. In many cases, students needed to combine several concepts and techniques from the course in the analysis of their roller coasters, helping them to see connections between various course topics. The project also gave students the chance to practice teamwork and time management skills.

\section{General Project Description}

To introduce the project, students were shown video of a roller coaster ride filmed from the first seat of an actual roller coaster followed by a simulated ride of the very same coaster as modeled in NoLimits. They were then informed that they would have the opportunity to use analytical and numerical methods to design a roller coaster and then simulate it in NoLimits to see first-hand how their design worked.

Specifically, each group of students was told that a local amusement park was soliciting proposals for the design of a new roller coaster. The park anticipated proposals from several designers, with the winning design offering the best value proposition (benefit for the cost). Thus, effort should be focused on determining a roller coaster track that provides the most thrilling yet safe and comfortable ride for the cost. Since passenger excitement, comfort, and safety are all related to the roller coaster's dynamics, in particular the forces acting on a roller coaster car as it travels down the track, determining suitable track geometry was the project's primary goal. No constraints on the design of the coaster itself were imposed, other than the geometric limits present within the NoLimits software, in order to give students the opportunity to pursue and justify their vision of the best roller coaster. 
Instruction regarding the use of NoLimits and regarding analysis appropriate to roller coaster design was provided throughout the semester. This was primarily done in short, fifteen minute mini-lectures during the fourth instructional hour of the week, although two regular lecture times were also used for this purpose. In addition, background readings covering the roller coaster design topics were available electronically. ${ }^{8-11}$

NoLimits was selected as the roller coaster simulation tool as it is the only commercial software available for this purpose that is also educationally licensed. Instruction regarding the use of NoLimits consisted of having students use the program's tutorial in the first two sessions and then answering specific student questions as they arose.

Student work on the coaster was assessed twice during the semester, once during the midterm period and once at the end of the semester. Details about the assessment are provided below.

\section{Project Resources}

Students were given the fourth instructional hour of the course each week to work on the project. However, on many occasions, part of this time was dedicated to a mini-lecture on a particular roller coaster topic or to short group meetings with the instructor to discuss particular track features or analysis/modeling skills. The techniques discussed on these occasions paralleled the supplement readings provided to the students. ${ }^{8-11}$ A short list of the concepts and techniques discussed includes:

- Defining g-force

- Analyzing coaster speed using energy methods

- Analyzing coaster normal force using free body diagrams

- Analyzing coaster transverse force for turns and banking

- Using Bezier curves to define geometry in NoLimits

- Using differential geometry to define hills, valleys, and loops for a specified g-force

- Solving differential equations using numerical methods in Excel and MATLAB

- Estimating roller coaster energy losses

- Heartlining roller coaster track

- Analyzing roller coaster corkscrews

In the first half of the course, students were provided with instruction on the concept of a g-force, the use of energy methods to determine roller coaster car velocity, and the use of free body diagrams to determine the normal force acting on a roller coaster car for 2-D track geometry. Example track used in this discussion included hills, valleys, linear sections at arbitrary angles, and circular loops. Students were required to examine the normal force acting on coaster cars at critical points such as hill crests, valley troughs, and the bottom, side, and top of a loop to determine the size and direction of the g-forces and how these relate to track curvature and roller coaster speed. Students were also provided with instruction on how to bank track to reduce transverse forces when considering turns. With this knowledge, they had to design and analyze an out-and-back roller coaster test track for their midterm assessment that was based on simple geometries such as circular arcs, straight lines, and banked turns. This helped ensure students had 
mastered the necessary basic analysis skills before tackling their final design. The test track also allowed students to practice putting geometry into the NoLimits program using Bezier curves, for which they were provided only simple rules at this stage.

In the second half of the course, students were encouraged to include more advanced features in their roller coasters, including loops, constant g-force drops and valleys, air-time hills, and corkscrews. All student groups included two or more of these features in their final roller coasters. With the exception of corkscrews, all of these features require the numerical solution of differential equations in order to determine the correct geometry. Students were provided instruction on how to solve these equations numerically using differencing methods in Excel and ode45 in MATLAB. Students were also presented with additional information about Bezier curves and introduced to a MATLAB package that could easily translate a set of geometry defining points to a simpler set of Bezier points. ${ }^{12}$ How to account for energy losses caused by rolling resistance and air drag, how to heartline track by basing coaster geometry on the path followed by riders' torsos rather than the path followed by the roller coaster track, and how to construct corkscrews were other subjects discussed during the second half of the project.

\section{Midterm Project Assessment}

For the midterm assessment, worth $5 \%$ of the course grade, students were asked to address three key questions with their deliverables:

- Is there a clear vision for the roller coaster design?

- Are the technical skills necessary to complete the design being developed?

- How can the time and resources remaining in the semester be budgeted to ensure a successful project outcome?

Each of these big picture questions was explored in more detail by individual deliverables: a project proposal, preliminary calculations, and reflections. No fixed format was specified for each of these deliverables, but certain items needed to be included.

In the project proposal, students were asked to document evidence of the plan they had developed for their final coaster via concise descriptions, annotated sketches/graphics, rough layouts in NoLimits, and notes they had made during the first half of the semester. At a minimum, they were expected to provide:

- A list of major roller coaster features and the order of their arrangement.

- A sketch or graphic showing the geometric layout of the major features.

- Reasoning behind early design choices such as what makes the designed coaster unique, exciting, and attractive and why the particular layout was chosen.

In the preliminary calculations, students were expected to present calculations for their out-andback test track involving hills, valleys, and banking constructed using geometric primitives. They had the goal of convincing an outside observer that they had the technical skills necessary to properly analyze their project. At a minimum, they were expected to provide: 
- Pencil and paper, Excel, or MATLAB calculations of the expected normal forces a roller coaster car would experience at key points along the test track geometry.

- Pencil and paper, Excel, or MATLAB calculations of the expected banking necessary for the test track geometry.

- A simulation of the track geometry within the NoLimits simulator corresponding to their calculations.

- A comparison between the analytical calculations and the simulation results from NoLimits considering the questions:

$\circ$ Do the calculations and simulation agree?

- Can any disagreement be quantified?

- What reasons might explain the disagreement between the calculations and simulation?

○ How could the hypothesized reasons for disagreement be tested?

In the reflections, students were asked to make a plan to best utilize the time and resources of the remainder of the semester by addressing the following points:

- What major questions do you need to answer before you can finish your design?

- What additional skills do you need to develop before you can finish your design?

- How will you budget your time over the coming weeks to ensure project completion?

- What project milestones do you expect to accomplish each week?

- How can you better leverage the human resources of your team?

- How will you ensure proper documentation in the final design portfolio?

- What have you learned from the project so far?

Each of these deliverables was graded on the basis of a five-point scale with possible grades assigned according to the following criteria:

- Exemplary: Analysis/commentary is complete. Mastery of the subject matter is displayed.

- Accomplished: Analysis/commentary is sufficient but with minor errors. Command of the subject matter is evident.

- Developing: Analysis/commentary is suitable but with items missing or some errors. Reasonable grasp of the subject matter is evident.

- Beginning: Analysis/commentary is uneven, with major omissions or significant errors. It is unclear if the subject matter is understood.

- Poor: Analysis/commentary is poor, entirely missing, or with egregious error. The subject matter is not understood.

Written feedback was provided to each team, with additional follow-up during the check-in sessions in class. The most significant area where students needed to better develop their thinking was regarding why they saw differences between their calculations and the simulation and how they might test hypotheses for any differences they observed. 


\section{Final Project Assessment}

For the final assessment, worth $10 \%$ of the course grade, students were also asked to address three key points with their deliverables that paralleled those in the midterm assessment:

- Share the final vision for your coaster in a way appropriate for a broad, public audience.

- Demonstrate the technical soundness of your design via engineering calculations and simulation.

- Reflect on what you have learned in terms of knowledge, skills, and intangibles from the project.

Each of these key points was developed in more detail by individual deliverables: promotional materials, engineering calculations, and reflections. Again, no fixed format was specified for each of these sections, but certain items needed to be included.

In the promotional materials, students were asked to document the final design of their roller coaster via visual and written descriptions. At a minimum, they were expected to provide:

- A roller coaster logo.

- A description of their coaster's major features, written in a way to attract riders.

- A computer graphic of their coaster showing its geometric layout with the major features labeled.

In the engineering calculations, students were expected to present the final calculations for their roller coaster design, with specific emphasis on demonstrating that the track geometry had been driven by the calculations as opposed to the calculations merely being in support of an arbitrary track. The minimum requirements for this section were the same as for the midterm review (although for the final track with more complicated features rather than the previous test track). Also, all of the calculations had to be Excel or MATLAB-based.

In the reflections, students were asked to think about and provide commentary on what they took away from the design and learning process. Specifically, the following prompts were provided:

- Which, if any, dynamics-related ideas/misconceptions did the project make real/clarify for you?

- Did the project help you develop thinking skills (problem solving, conceptualization, etc.)?

- Did the project help you develop engineering skills (solving ODEs, using Bezier curves, etc.)?

- Did the project help you develop interpersonal skills (working in a team, budgeting time, etc.)?

- Did your perspective on the engineering of amusement rides change over the course of the project?

- If you had to start the project over again, what would you do differently?

- What is your biggest takeaway from the project? 
Questions similar to these reflection questions were also present on end-of-term student feedback forms.

The grading scale employed in reviewing the final project assessments mirrored that used for the midterm project assessments. Overall, the students did excellent work on the project and developed roller coasters that were good first approximations to something that might be realized at a theme park. An example of a student-designed coaster is shown in Figure 1.

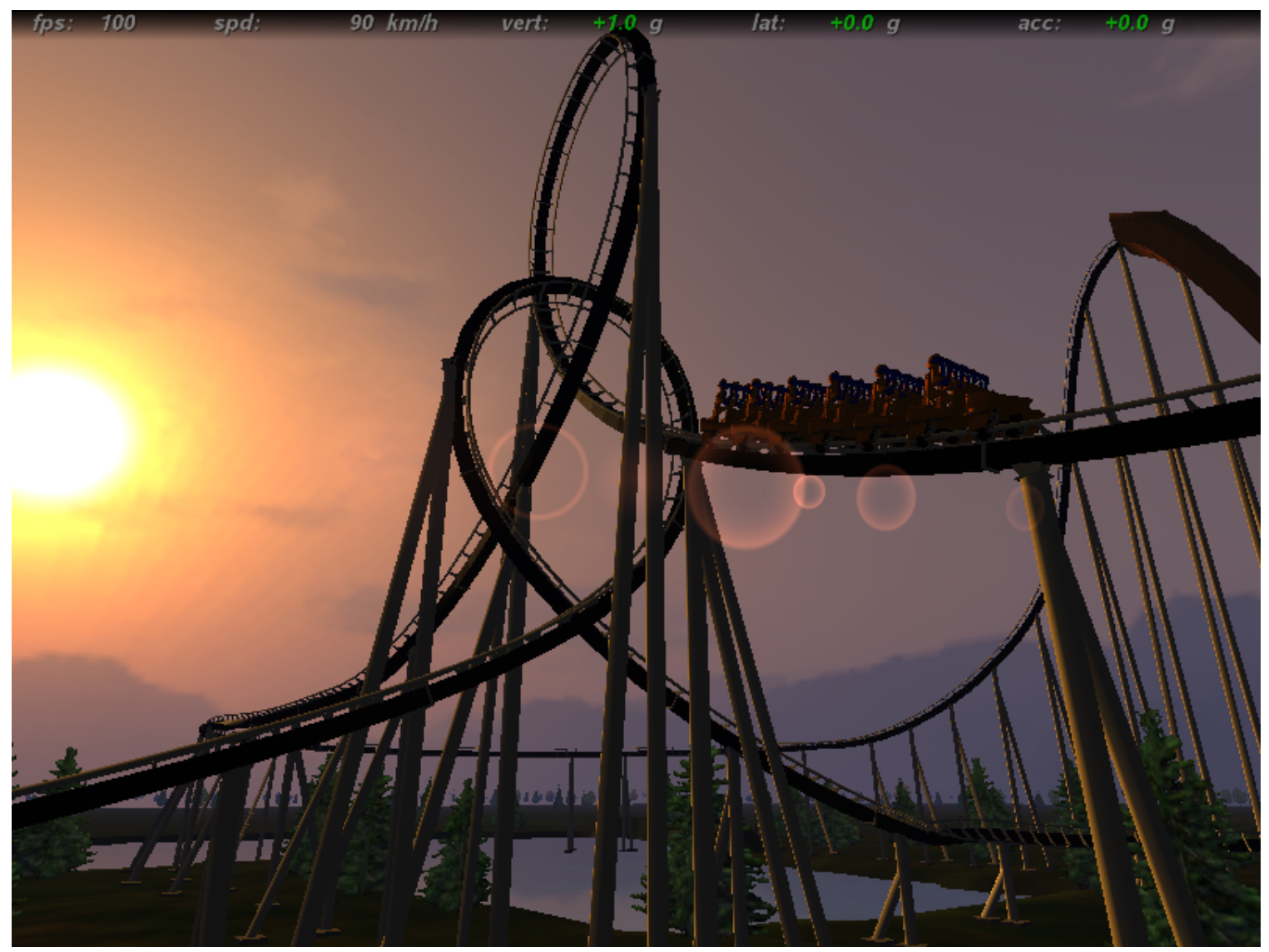

Figure 1: A student-designed roller coaster as rendered in the NoLimits software.

\section{Student Feedback}

At the end of the project, students were asked to provide feedback about the project via a set of questions rating certain aspects on a five-point scale and via questions open to free-form comments. The feedback form used is documented in Appendix A. Twenty-one of the twentyfour students enrolled in the class completed a form. Overall, the students responded very favorably to the project and felt it helped them better understand concepts from the course as well as developing analysis and teamwork/time management skills.

Figure 2 shows student responses to questions focused on whether the project helped them learn specific concepts and skills related to dynamics. In general, the students strongly agreed that the project helped them better appreciate basic practices such as free body diagrams and energy calculations and that it also gave them a better appreciation of the forces involved in roller 
coasters. Some of the more advanced skills, such as using MATLAB to solve ordinary differential equations and to approximate geometry using Bezier curves, saw slightly less perceived student learning, although the majority of students still agreed they had developed some skills in these areas. What is most exciting about the results in this section, however, is that every student agreed, with over three quarters agreeing strongly, that the project had helped them see how dynamics could be used in a design setting. This strongly supports the idea that the project is a powerful vehicle for helping students see dynamics in a different context within engineering.

In Figure 3, student perceptions of how the project helped them with teamwork and time management skills are presented. In general students agreed that the project had helped them with these skills, although no specific instruction was provided on these issues.

Finally, in Figure 4 some results regarding student evaluation of project expectations, resources, and workload are provided in addition to how much students enjoyed working on the project. Generally, students found the project to be clearly laid out and the workload reasonable, with most students reporting an enjoyable experience.

Some of the comments from the written feedback also support the previous assertions. Regarding how the project spurred learning by making the use of dynamics fun, one student commented, "The roller coaster was a fun way to apply what we learned during the semester and (was) really enjoyed by everyone, so I felt the students did not treat the project as an obligation..." The students also generally picked up that using dynamics for practical problems was often complex and that assumptions are very important, with one student stating, "Real life systems are COMPLEX - any assumptions you make may simplify the analysis BUT it also causes deviations from actual behavior." In one of the final assessment documents another set of students wrote:

...problem solving skills and troubleshooting became a major part of this project when we hit multiple dead ends and had predictions that didn't match with the simulator. Every time there was an inconsistency between the model and our calculations we went back and made a conjecture about what could be causing the difference.

Yet another set of students wrote this synopsis:

In our opinion, the biggest takeaway from this project was acknowledging and coping with the sheer amount of information, assumptions and details that "real-world" engineers do day in and day out. It is hard to appreciate the thought that goes into such a large-scale project until one is handed to you. What is even more mind-boggling is that our analysis was purely from an engineering standpoint; economic, geographic and many other considerations were not taken into account.

These types of broad lessons about using concepts from dynamics in engineering practice are not easily grasped via normal textbook problem solving, but become apparent via this project-based approach. 
$\mathbf{A}$

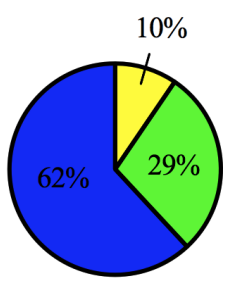

This project helped me understand and visualize the forces involved in a roller coaster.

D

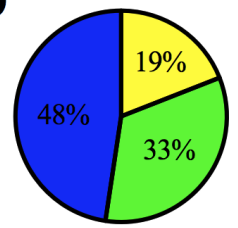

This project helped me understand the importance of using free body and kinetic diagrams to analyze particle motion.

G

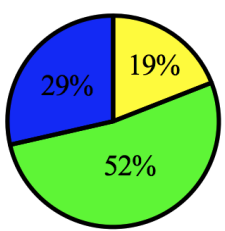

This project helped me develop technical skills for numerically solving ODEs using MATLAB or Excel.
B

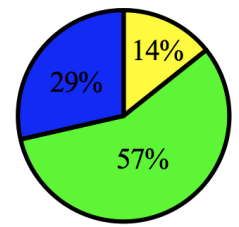

This project helped me understand normal and tangential acceleration.

$\mathbf{E}$

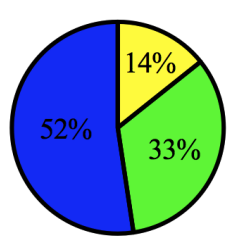

This project helped me understand how to use kinetic and potential energy to determine particle velocity in a conservative system.

H

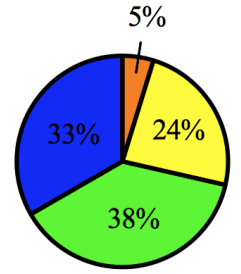

This project helped me develop technical skills for approximating geometry using Bezier curves.
C

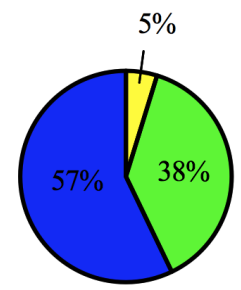

This project helped me understand what is meant by a gforce.

F

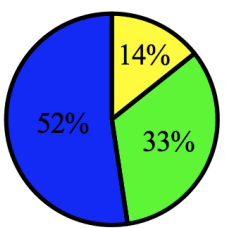

This project helped me understand the need to consider energy losses in real systems.

I

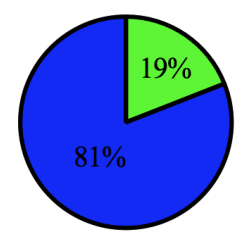

This project helped me see how dynamics could be used in a design setting.

Figure 2: Evaluation of the project's success in helping students understand dynamics concepts and skills. Evaluation was based on student responses to the displayed statements in regard to forces (A), types of acceleration (B), g-force (C), using free body and kinetic diagrams (D), using kinetic and potential energy calculations (E), considering energy losses (F), numerically solving ODEs $(\mathrm{G})$, using Bezier curves $(\mathrm{H})$, and using dynamics in a design setting (I). 


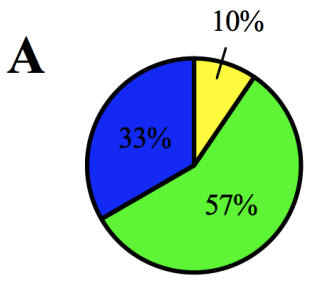

This project helped me develop teamwork skills.
B

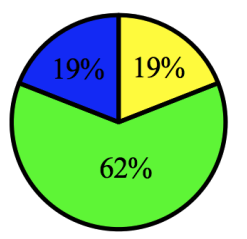

This project helped me learn to organize tasks and budget time.

Figure 3: Evaluation of the project's success in helping students develop teamwork and organizational skills. Evaluation was based on student responses to the displayed statements in regard to teamwork skills (A) and organizational skills (B).

$\mathbf{A}$

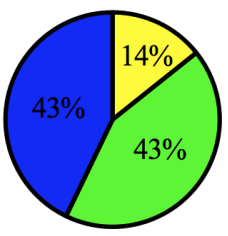

The expectations for the project deliverables were clear.

C

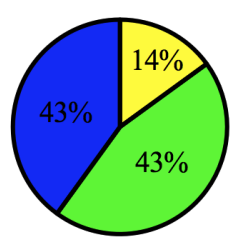

The workload for the project was reasonable.

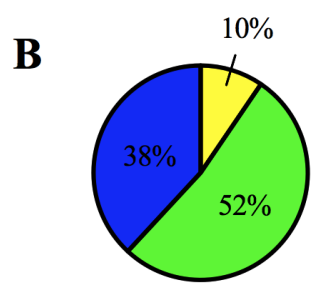

The information provided by the instructor was sufficient for completing the project.

Strongly Agree

Agree

Neutral

Disagree

Strongly Disagree

Figure 4: Evaluation of the execution of the project and overall student experience. Evaluation was based on student responses to the displayed statements in regard to clarity of expectations (A), sufficiency of provided information (B), project workload (C), and student enjoyment (D). 


\section{Future Improvements}

Several students commented that they wished the roller coaster project had several smaller but more frequent deliverables to keep them on track. They felt they wasted time early in the semester playing with NoLimits rather than developing the skills necessary to analyze their roller coaster design. While this may indicate a lesson about time management has been learned, it would be beneficial to include two interim deliverables to ensure all students are taking the steps necessary to produce a successful design. A guide to the NoLimits program and some of its idiosyncrasies might also be helpful to aid acclimatization to the software.

Students also commented that they wished the requirements for the deliverables were more clear and available at the beginning of the semester. For future versions of the project, all materials regarding deliverables as well as the grading scale would be made available to the students at the beginning of the semester rather than presented piecemeal.

This project scales easily, and it would have been beneficial to expose students to some of the standards used in roller coaster design. ${ }^{13}$ In future iterations of the project, it would be interesting to explore working standards into the project materials, although the workload is already heavy if only one hour each week is allotted to the project.

\section{Conclusions}

Incorporating a roller coaster project into a first course in engineering dynamics improves students' learning experiences. Dynamics is cast in a design context and connected to a real world problem, which motivates students to learn the material. This allows different aspects of dynamics to be emphasized, particularly the choices involved in creating mathematical models of physical systems. Such a project also allows students to make connections between various dynamics concepts, reinforces the problem solving and analysis techniques developed in class, and offers an opportunity to exercise creativity and good design practices while working on a team.

\section{Acknowledgements}

The project was partially supported by the Kern Entrepreneurial Engineering Network (KEEN). Thanks to Dr. Krousgrill and Dr. Rhoads at Purdue University for discussions about their roller coaster design class ME 297: Roller Coaster Dynamics.

\section{References}

[1] Everett, L. J., 1997, "Dynamics as a process, helping undergraduates understand design and analysis of dynamic systems,” Proc. 118th ASEE Annual Conference, Milwaukee, WI, pp. 2666.

[2] Mikesell, D. R. and Yoder, J. S., 2011, “Teaching dynamics with a design projects,” Proc. 122nd ASEE Annual Conference, Vancouver, BC, pp. AC 2011-615. 
[3] Jolley, W., Rencis, J., Cobb, E. and Haggland, R., 1997, "Incorporating a LEGO fourbar mechanism project in undergraduate dynamics," Proc. 32nd ASEE/IEEE Frontiers in Education Conference, Boston, MA.

[4] Birdsong, C., 2012, "Using automotive safety in a service-learning project for undergraduate dynamics," Proc. 119th ASEE Annual Conference, San Antonio, TX.

[5] Personal communication with Krousgrill, C.M. and Rhoads, J. regarding the course ME 297: Roller Coaster Dynamics at Purdue University.

[6] Wick, D. P., Ramsdell, M. W., Fowler, K., Turner, P., and Schalk, P. D., 2011, "University Outreach in STEM Education through a Roller Coaster Science and Engineering Camp," Proc. 41st ASEE/IEEE Frontiers in Education Conference, Rapid City, SD, pp. S3D- 1-6.

[7] Hibbeler, R. C., 2012, Engineering Mechanics: Dynamics, Prentice Hall, Cambridge, UK, Chap. 6.

[8] Clarke, A., Jr., 1988, “A Primer on Roller Coaster Dynamics Part I - Plane and Fancy,” RollerCoaster!, 9(3-4), pp. 30-37.

[9] Clarke, A., Jr., 1989, “A Primer on Roller Coaster Dynamics Part II - You Can Bank on Them,” RollerCoaster!, 10(1), pp. 32-37.

[10] Clarke, A., Jr., 1989, “A Primer on Roller Coaster Dynamics Part III - Passengers Entrained,” RollerCoaster!, 10(2), pp. 24-29.

[11] Pendrill, A., 2005, “Rollercoaster loop shapes,” Phys. Educ., 40(6), pp. 517-521.

[12] Khan, M., 2007, “Cubic Bezier Least Square Fitting”, from

http://www.mathworks.com/matlabcentral/fileexchange/15542-cubic-bezier-least-square-fitting.

[13] ASTM, 2012, “ASTM Standards on Amusement Rides and Devices $8^{\text {th }}$ Edition”, RIDES12. 


\section{Appendix A: Roller Coaster Project Feedback Form}

Please answer each question as it pertains to the roller coaster project.

The project helped me understand and visualize the forces involved in a roller coaster.

The project helped me understand normal and tangential acceleration.

The project helped me understand what is meant by a g-force.

The project helped me understand the importance of using free body and kinetic diagrams to analyze particle motion.

The project helped me understand how to use kinetic and potential energy to determine particle velocity in a conservative system.

The project helped me understand the need to consider energy losses in real systems.

The project helped me see how dynamics could be used in a design setting.

The project helped me develop technical skills fo numerically solving ODEs using MATLAB or Excel.

The project helped me develop technical skills for approximating geometry using Bezier curves.

The project helped me develop team work skills.

The project helped me learn to organize tasks and budget time.

The expectations for the project deliverables were clear.

The information provided by the instructor was sufficient for competing the project.

The workload for the project was reasonable.

I enjoyed working on the project.

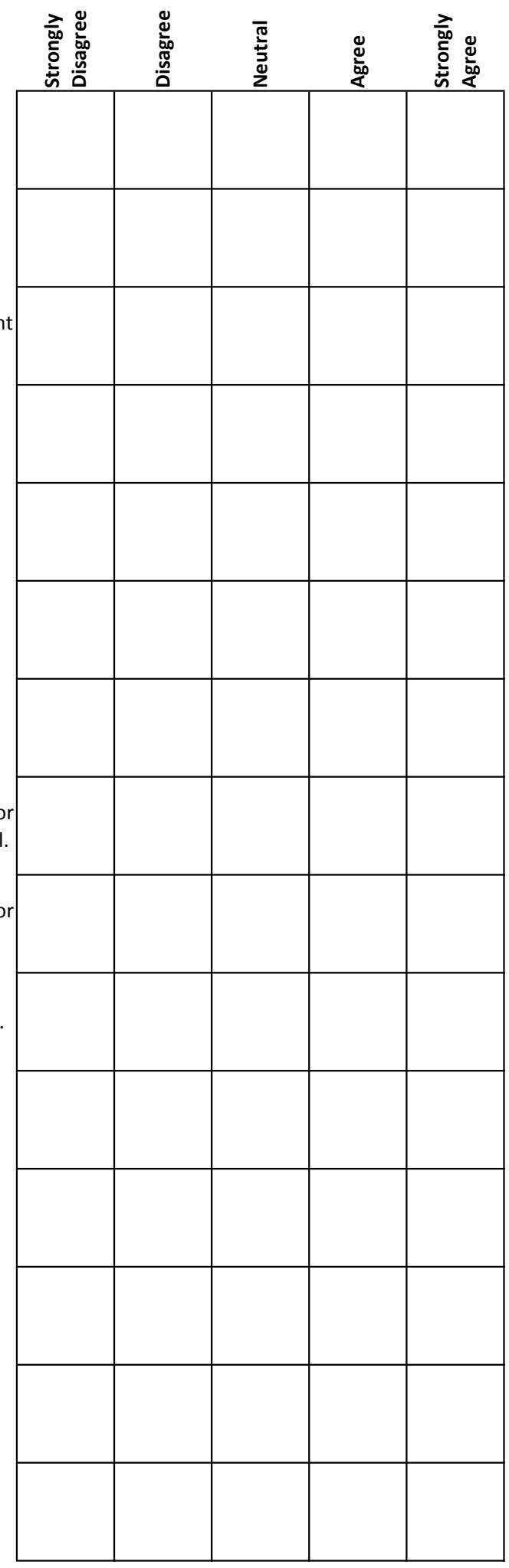


1. What part of the project did you enjoy the most?

2. What is the most significant thing you learned from the project?

3. What aspect of the project could be improved? How would you make an improvement?

4. Other project related comments. 


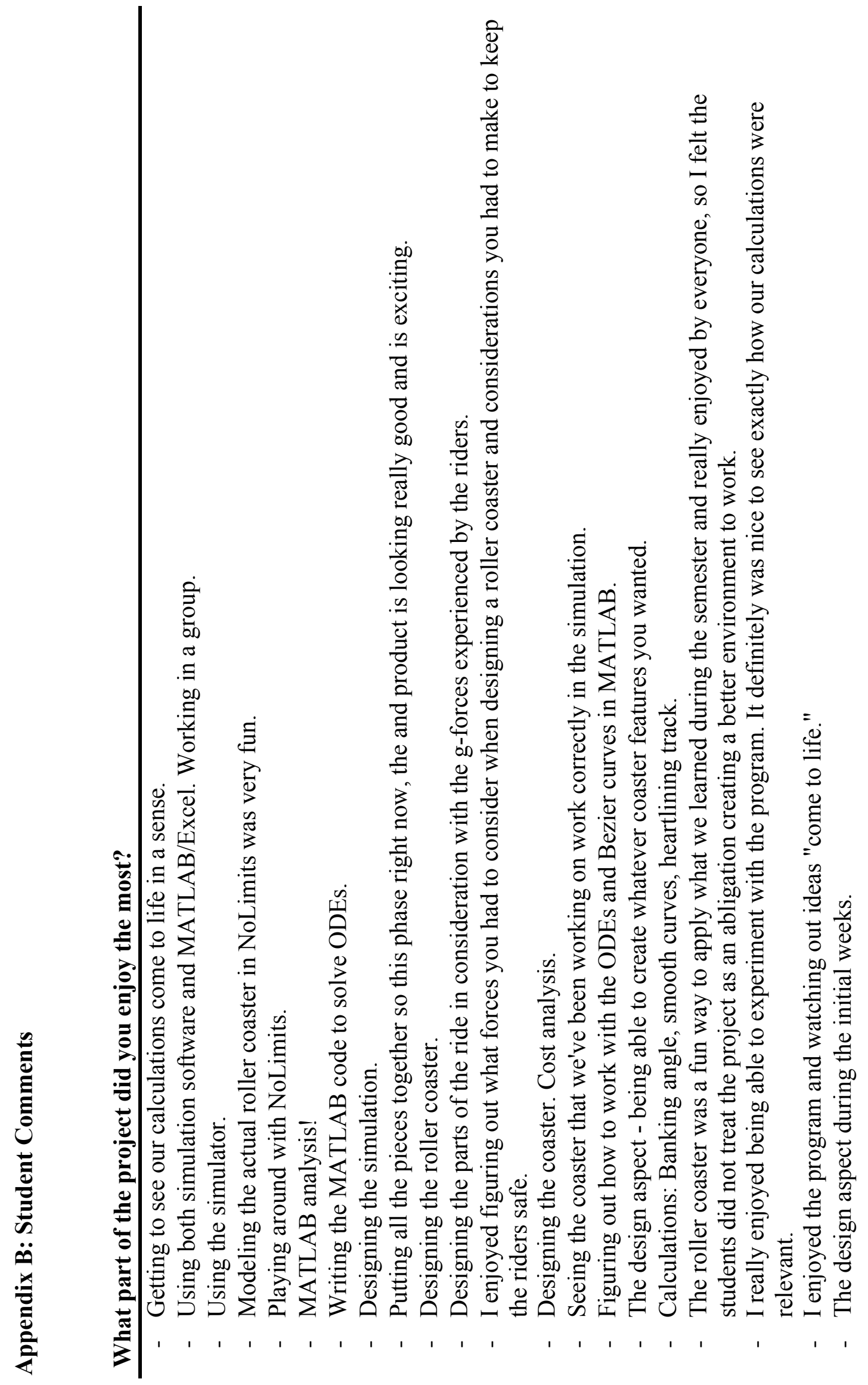




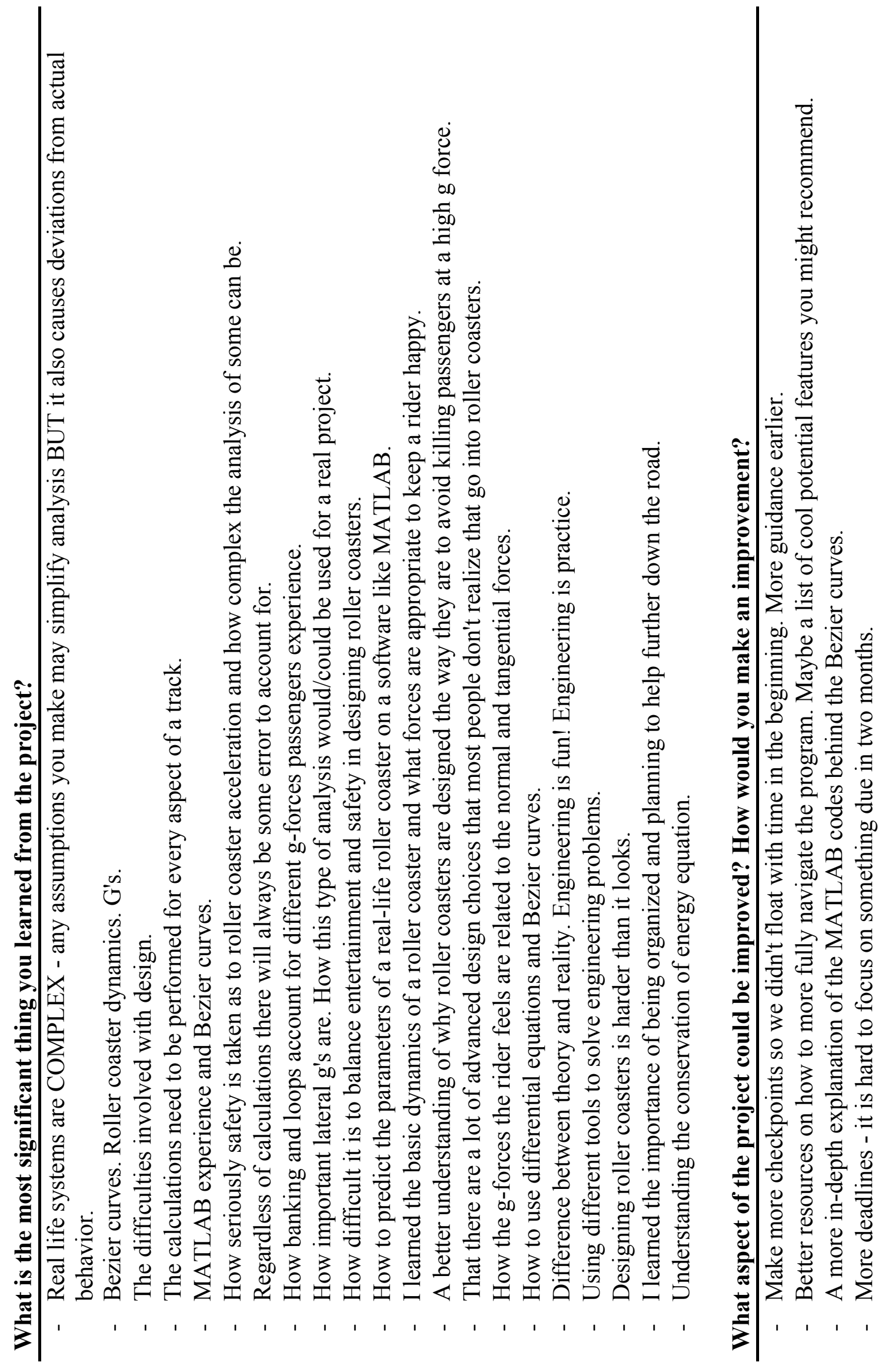




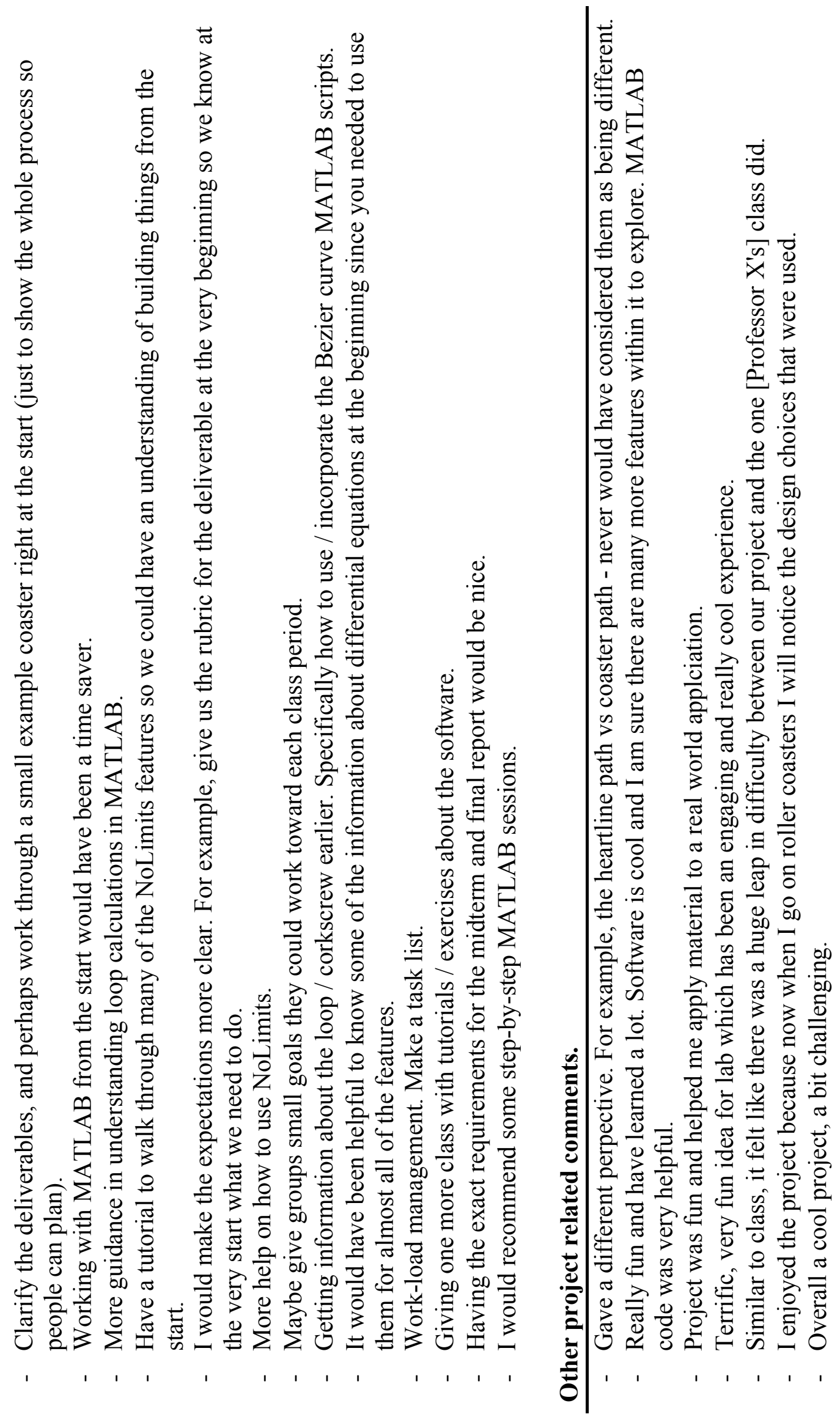

0
$\mathscr{0}$
0
$N$
0
$\stackrel{0}{0}$
$\stackrel{+}{0}$
$\infty$ 